ARTICLE

Received 4 Dec 2015 | Accepted 5 May 2016 | Published 13 Jun $2016 \quad$ DOl: 10.1038/ncomms11839 $\quad$ OPEN

\title{
Arrays of individually controlled ions suitable for two-dimensional quantum simulations
}

Manuel Mielenz ${ }^{1}$, Henning Kalis ${ }^{1}$, Matthias Wittemer ${ }^{1}$, Frederick Hakelberg ${ }^{1}$, Ulrich Warring ${ }^{1}$, Roman Schmied ${ }^{2}$, Matthew Blain ${ }^{3}$, Peter Maunz ${ }^{3}$, David L. Moehring ${ }^{3, \dagger}$, Dietrich Leibfried ${ }^{4}$ \& Tobias Schaetz ${ }^{1,5}$

A precisely controlled quantum system may reveal a fundamental understanding of another, less accessible system of interest. A universal quantum computer is currently out of reach, but an analogue quantum simulator that makes relevant observables, interactions and states of a quantum model accessible could permit insight into complex dynamics. Several platforms have been suggested and proof-of-principle experiments have been conducted. Here, we operate two-dimensional arrays of three trapped ions in individually controlled harmonic wells forming equilateral triangles with side lengths 40 and $80 \mu \mathrm{m}$. In our approach, which is scalable to arbitrary two-dimensional lattices, we demonstrate individual control of the electronic and motional degrees of freedom, preparation of a fiducial initial state with ion motion close to the ground state, as well as a tuning of couplings between ions within experimental sequences. Our work paves the way towards a quantum simulator of two-dimensional systems designed at will.

\footnotetext{
${ }^{1}$ Albert-Ludwigs-Universität Freiburg, Physikalisches Institut, Hermann-Herder-Strasse 3, Freiburg 79104, Germany. ${ }^{2}$ Department of Physics, University of Basel, Klingelbergstrasse 82, Basel 4056, Switzerland. ${ }^{3}$ Sandia National Laboratories, PO Box 5800 Albuquerque, New Mexico 87185-1082, USA. ${ }^{4}$ Time and Frequency Division, National Institute of Standards and Technology, 325 Broadway, Boulder, Colorado 80305, USA. ${ }^{5}$ Albert-Ludwigs-Universität Freiburg, Freiburg Institute for Advanced Studies, Albertstr. 19, 79104 Freiburg, Germany. † Present address: The Intelligence Advanced Research Projects Activity, College Park, MD, USA. Correspondence and requests for materials should be addressed to U.W. (email: ulrich.warring@physik.uni-freiburg.de).
} 
$\mathrm{R}$ ichard Feynman was one of the first to recognize that quantum systems of sufficient complexity cannot be simulated on a conventional computer ${ }^{1}$. He proposed to use a quantum mechanical system instead. A universal quantum computer would be suitable, but practical implementations are a decade away at best. However, universality is not required to simulate specific quantum models. It is possible to custom-build an analogue quantum simulator (AQS) that allows for preparation of fiducial input states, faithful implementation of the model-specific dynamics and for access to the crucial observables. Simulations on such AQSs could impact a vast variety of research fields ${ }^{2}$, that is, physics ${ }^{3}$, chemistry ${ }^{4}$ and biology ${ }^{5}$, when studying dynamics that is out of reach for numerical simulation on conventional computers.

Many experimental platforms have been suggested to implement AQSs ${ }^{6-9}$. Different experimental systems provide certain advantages in addressing different physics. Results that are not conventionally tractable may be validated by comparing results of different AQSs simulating the same problem ${ }^{10,11}$. Over the last two decades, many promising proof-of-principle demonstrations have been made using photons ${ }^{6}$, superconductors ${ }^{7}$, atoms ${ }^{8}$ and trapped atomic ions 9 . Trapped ions in particular have seen steady progress from demonstrations with one or two ions ${ }^{12-18}$ to addressing aspects of quantum magnets ${ }^{19}$ with linear strings of $2-16$ ions ${ }^{13,20}$ and self-ordered two-dimensional crystals containing more than 100 ions $^{21}$. Ions are well suited to further propel the research since they provide long-range interaction and individual, fast controllability with high precision ${ }^{22}$.

Two-dimensional trap-arrays may offer advantages over trapping in a common potential, because they are naturally suited to implement tuneable couplings in more than one spatial dimension. Such couplings are, in most cases, at the heart of problems that are currently intractable by conventional numerics ${ }^{10,23}$. Our approach is based on surface-electrode structures ${ }^{24}$ originally developed for moving ion qubits through miniaturized and interconnected, linear traps as proposed in refs 25,26 . This approach is pursued successfully as a scalable architecture for quantum computer, see, for example, ref. 27. For AQSs, it is beneficial to have the trapped ion ensembles coupled all-to-all so they evolve as a whole. This is enabled by our array architecture with full control over each ion. Individual control allows us to maintain all advantages of single trapped ions while scaling the array in size and dimension ${ }^{28-30}$.

Optimized surface electrode geometries can be found for any periodic wallpaper group as well as quasi-periodic arrangements, as, for example, Penrose-tilings ${ }^{29}$. A first step, trapping of ions in two-dimensional arrays of surface traps, has been proposed ${ }^{15}$ and demonstrated $^{31}$. Boosting the strength of interaction to a level comparable to current decoherence rates requires inter-ion distances $d$ of a few tens of micrometres. Such distances have been realized in complementary work, where two ions have been trapped in individually controlled sites of a linear surfaceelectrode trap at $d$ between 30 and $40 \mu \mathrm{m}$. The exchange of a single quantum of motion, as well as entangling spin-spin interactions have been demonstrated in this system ${ }^{32,33}$. The increase in coupling strength was achieved with a reduction of the ion-surface separation to order $d$ and the concomitant increase in motional heating due to electrical noise. Recently, methods for reducing this heating by more than two orders of magnitude with either surface treatments ${ }^{34-36}$ or cold electrode surfaces ${ }^{37-39}$ have been devised.

Here, we demonstrate the precise tuning of all relevant parameters of a two-dimensional array of three ions trapped in individually controlled harmonic wells on the vertices of equilateral triangles with side lengths 80 and $40 \mu \mathrm{m}$. In the latter, Coulomb coupling rates ${ }^{32}$ approach current rates of decoherence. Dynamic control permits to reconfigure Coulomb and laser couplings at will within single experiments. We initialize fiducial quantum states by optical pumping, Doppler and resolved sideband cooling to near the motional ground state. Our results demonstrate important prerequisites for experimental quantum simulations of engineered twodimensional systems.

\section{Results}

Trap arrays and control potentials. Our surface ion trap chip is fabricated in similar manner to that described in ref. 40 and consists of two equilateral triangular trap arrays with side length of $\simeq 40$ and $\simeq 80 \mu \mathrm{m}$, respectively (Fig. 1a,b), both with a distance of $\simeq 40 \mu \mathrm{m}$ between the ions and the nearest electrode surface. The shapes of radio-frequency (RF) electrodes of the arrays are optimized by a linear-programming algorithm that yields electrode shapes with low fragmentation, and requires only a single RF-voltage source for operation ${ }^{29,30}$. To design different and even non-periodic arrays for dedicated trap distances, we can apply the same algorithm to yield globally optimal electrode shapes $^{29}$. Resulting electrode shapes may look significantly different, but will have comparable complexity, spatial extent and the same number of control electrodes per trap site. Therefore, we expect that different arrays will not require different fabrication techniques (Methods). The two arrays are spaced by $\simeq 5 \mathrm{~mm}$ on the chip, and only one of them is operated at a given time. Although we achieve similar results in both arrays, the following discussion is focussed on the $80 \mu \mathrm{m}$ array.

Three-dimensional confinement of ${ }^{25} \mathrm{Mg}^{+}$ions is provided by a potential $\phi_{\mathrm{RF}}$ oscillating at $\Omega_{\mathrm{RF}}$ from a single RF electrode driven at $\Omega_{\mathrm{RF}} /(2 \pi)=48.3 \mathrm{MHz}$ with an approximate peak voltage $U_{\mathrm{RF}}=20 \mathrm{~V}$. Setting the origin of the coordinate system at the centre of the array and in the surface plane of the chip, the RF potential features three distinct trap sites at $\mathbf{T} \mathbf{0} \simeq(-46,0,37) \mu \mathrm{m}$, $\mathbf{T} 1 \simeq(23,-23 \sqrt{3}, 37) \mu \mathrm{m}, \quad$ and $\quad \mathbf{T} 2 \simeq(23,23 \sqrt{3}, 37) \mu \mathrm{m}$. Owing to the electrode symmetry under rotations of $\pm 2 \pi / 3$ around the $z$-axis, it is often sufficient to consider T0 only, as all our findings apply to $\mathbf{T} 1$ and $\mathbf{T} 2$ after an appropriate rotation. Further, the RF potential exhibits another trap site at $\simeq(0,0,81) \mu \mathrm{m}$ (above the centre of the array); this 'ancillary' trap is used for loading as well as for re-capturing ions that escaped from the other trap sites. We approximate the RF confinement at position $\mathbf{r}$ by a pseudopotential $\phi_{\mathrm{ps}}(\mathbf{r})=\mathrm{Q} /\left(4 m \Omega_{\mathrm{RF}}^{2}\right) E_{\mathrm{RF}}^{2}(\mathbf{r}), \mathrm{cp}$. ref. 41 , where $Q$ denotes the charge and $m$ the mass of the ion, and $E_{\mathrm{RF}}(\mathbf{r})$ is the field amplitude produced by the electrode. Calculations of trapping potentials are based on ref. 42 and utilizing the software package ${ }^{43}$. Equipotential lines of $\phi_{\mathrm{ps}}$ are shown in Fig. 1c-e.

Near T0 we can approximate $\phi_{\mathrm{ps}}$ up to second order and diagonalize the local curvature matrix to find normal modes of motion described by their mode vectors $\mathbf{u}_{1}, \mathbf{u}_{2}$ and $\mathbf{u}_{3}$, which coincide (for the pure pseudopotential) with $x, y$ and $z$; we use $\mathbf{u}_{j}$ with $j=\{1,2,3\}$ throughout our manuscript to describe the mode vectors of a single ion near To. We find corresponding potential curvatures of $\kappa_{\mathrm{ps}, 1} \simeq 3.0 \times 10^{8} \mathrm{~V} \mathrm{~m}^{-2}, \kappa_{\mathrm{ps}, 2} \simeq 5.9 \times 10^{7} \mathrm{~V} \mathrm{~m}^{-2}$ and $\kappa_{\mathrm{ps}, 3} \simeq 9.2 \times 10^{7} \mathrm{~V} \mathrm{~m}^{-2}$, whereas mode frequencies can be inferred from these curvatures as $\omega_{j} \simeq \sqrt{(Q / m) \kappa_{\mathrm{ps}, j}}$, with $j=\{1,2,3\}$ : $\omega_{1} /(2 \pi) \simeq 5.4 \mathrm{MHz}, \quad \omega_{2} /(2 \pi) \simeq 2.4 \mathrm{MHz}$ and $\omega_{3} /(2 \pi) \simeq 3.0 \mathrm{MHz}$. Further, the Mathieu parameters $q_{i}=2(Q / m) U_{\mathrm{RF}} / \Omega_{\mathrm{RF}}^{2} \kappa_{\mathrm{RF}, \mathrm{i}}(\mathbf{r})$, where $\kappa_{\mathrm{RF}, \mathrm{i}}(\mathbf{r})$ denotes the curvature of $\phi_{\mathrm{RF}}$ along direction $i=\{x, y, z\}$, at T0 are: $q_{x} \simeq-0.32, q_{y} \simeq 0.14$ and $q_{z} \simeq 0.18$.

To gain individual control of the trapping potential at each site, it is required to independently tune local potentials near T0, T1 and T2 (Methods), that is, to make use of designed local electric 
a

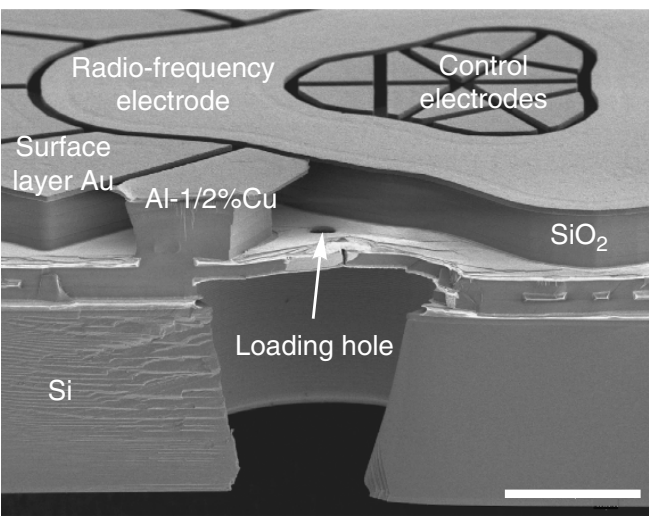

C

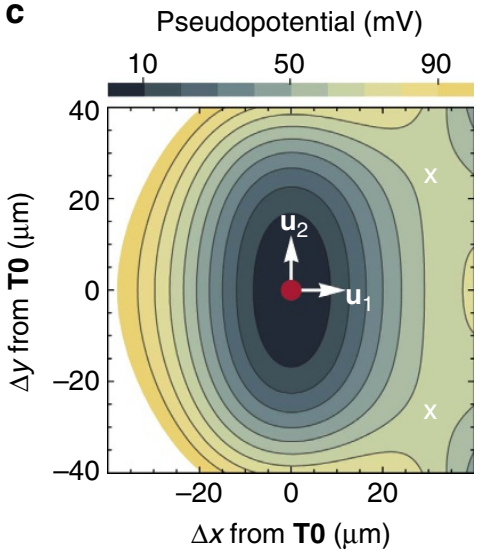

d

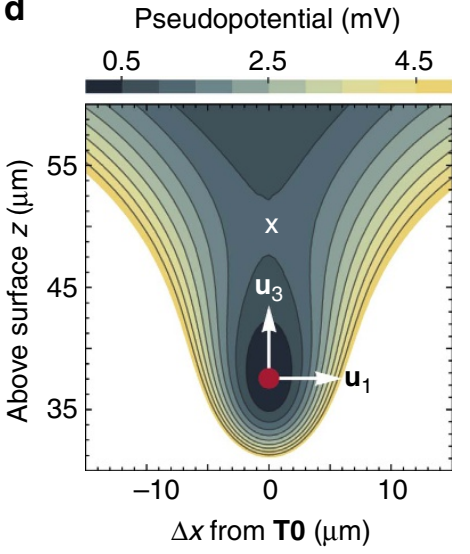

b

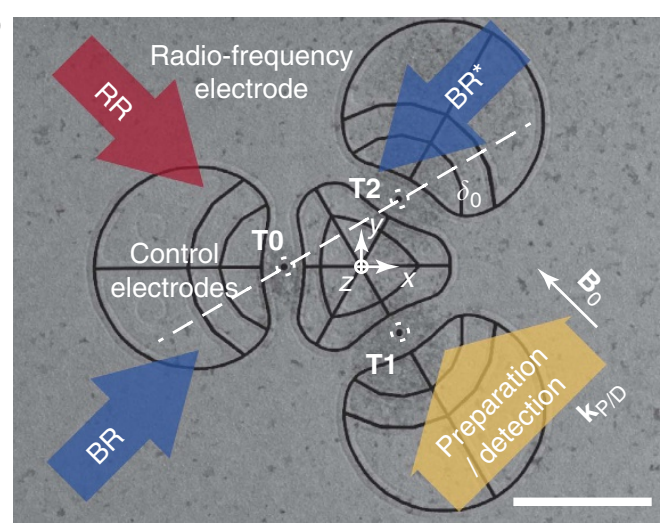

e Pseudopotential $(\mathrm{mV})$

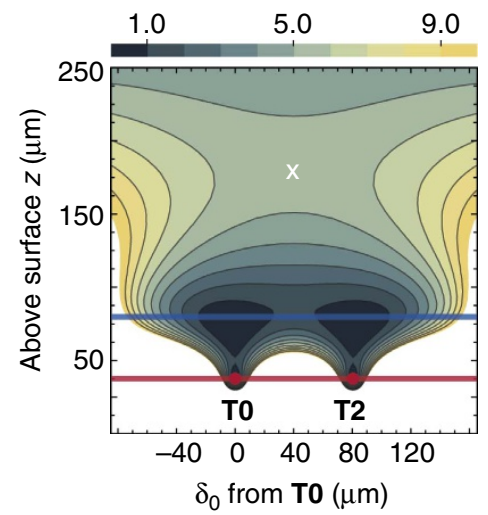

Figure 1 | Surface-electrode ion trap featuring three individual traps. (a) Scanning electron microscope (SEM) image of a cleaved copy of our chip; white scale bar, $20 \mu \mathrm{m}$. It provides a cross-sectional view vertically through the trap chip (bottom half of image) and a top view of the horizontal, planar trap electrode surface (top half of image) of the $40 \mu \mathrm{m}$ array. Buried electrode interconnects as well as the overhangs of electrodes that shield trapped ions from insulating surfaces are exposed in this view. A loading channel, vertically traversing the chip, collimates a neutral atom beam from an oven on the backside of the chip. (b) SEM top-view of the $80 \mu \mathrm{m}$ array, dark lines indicate gaps between individual electrodes and dashed circles highlight the three trap sites at TO, T1 and $\mathbf{T 2}$ that lie $40 \mu \mathrm{m}$ above the electrode plane (white scale bar, $80 \mu \mathrm{m}$ ); corresponding loading holes appear as dark spots. A vertical plane connecting TO and $\mathbf{T 2}$ is shown as a dotted line and labelled with $\delta_{0}$. The single RF electrode extends beyond the image area and encloses 30 control electrodes grouped into four islands depicted in the central part of the image, enabling the control of individual trap sites. Laser beams (coloured arrows) are parallel to the chip surface and wave vectors $\mathbf{k}_{\mathrm{P} / \mathrm{D}}$ of preparation and detection beams are parallel to the magnetic quantization field $\mathbf{B}_{0}$ (white arrow). (c-e) The pseudopotential $\phi_{\mathrm{ps}}(x, y, z)$ of the $80 \mu \mathrm{m}$ array in different planes is shown, trap sites marked by red dots, motional mode vectors $\mathbf{u}_{j}$ with $j=\{1,2,3\}$ at $\mathbf{T O}$ are represented by white arrows, and saddle points are illustrated by white crosses. In $\mathbf{e}$, the heights of T0, T2 and the ancillary trap are indicated by red and blue lines, respectively.

fields and curvatures. To achieve this, we apply sets of control voltages to 30 designated control electrodes (see Fig. 1 for details). In the following, a control voltage set is described by a unit vector $\hat{\mathbf{v}}_{\mathbf{c}} \equiv\left(\hat{v}_{\mathrm{c}, 1}, \ldots, \hat{v}_{\mathrm{c}, 30}\right)$, with corresponding dimensionless entries $\hat{v}_{c, n}$ with $n=\{1, \ldots, 30\}$, and result in a dimensionless control potential

$$
\hat{\phi}_{\mathrm{c}}=\sum_{n=1}^{30} \hat{v}_{\mathrm{c}, n} \hat{\phi}_{n}(\mathbf{r}),
$$

where $\hat{\phi}_{n}(\mathbf{r})$ is the potential resulting when applying $1 \mathrm{~V}$ to the $n$ th electrode following a basis function method ${ }^{44,45}$. We scale $\hat{\phi}_{\mathrm{c}}$ by varying a control voltage $U_{\mathrm{c}}$ and yielding a combined trapping potential

$$
\phi(\mathbf{r})=\phi_{\mathrm{ps}}(\mathbf{r})+U_{\mathrm{c}} \hat{\phi}_{\mathrm{c}}(\mathbf{r}) .
$$

Bias voltages applied to the control electrodes are, in turn, fully described by $\mathbf{U}_{c}=U_{c} \hat{\mathbf{v}}_{c}$.
To design a specific $\hat{\phi}_{\mathrm{c}}$, we consider the second order Taylor expansion for a point $\mathbf{r}_{0}$ and small displacements $\Delta \mathbf{r}$ :

$$
\begin{aligned}
\hat{\phi}_{c}\left(\mathbf{r}_{0}+\Delta \mathbf{r}\right) & \simeq \hat{\phi}_{c}\left(\mathbf{r}_{0}\right)+\left.\left[\partial_{k}\right]^{\mathbf{T}} \hat{\phi}_{c}(\mathbf{r})\right|_{\mathbf{r}=\mathbf{r}_{0}} \cdot \Delta \mathbf{r} \\
& +\left.\frac{1}{2} \Delta \mathbf{r}^{\mathbf{T}} \cdot\left[\partial_{k} \partial_{l}\right] \hat{\phi}_{c}(\mathbf{r})\right|_{\mathbf{r}=\mathbf{r}_{0}} \cdot \Delta \mathbf{r}
\end{aligned}
$$

where $\left.\left[\partial_{k}\right]^{\mathbf{T}} \hat{\phi}_{\mathrm{c}}(\mathbf{r})\right|_{\mathbf{r}=\mathbf{r}_{0}}$ is the local gradient and $\left.\left[\partial_{k} \partial_{l}\right] \hat{\phi}_{c}(\mathbf{r})\right|_{\mathbf{r}=\mathbf{r}_{0}}$ is the traceless and symmetric matrix with indices $k$ and $l=\{x, y, z\}$ that describes the local curvature; square brackets denote vectors/matrices, $\partial$ partial derivatives and the superscript $\mathbf{T}$ the transpose of a vector. We constrain local gradients in their three degrees of freedom (DoF) and local curvatures in their five DoF at T0, T1 and T2, and solve the corresponding system of 24 linear equations to yield $\hat{\mathbf{v}}_{\mathrm{c}}$. In principle, it would be sufficient to use 24 control electrodes, however, we consider all electrodes and use the extra DoF to minimize the modulus of the voltages we need to apply for a given effect. 
In particular, we distinguish two categories of control potentials, denoted by $\hat{\varepsilon}$ and $\hat{\kappa}$, respectively: the first category is designed to provide finite gradients and zero curvatures at T0, with zero gradients and curvatures at $\mathbf{T} 1$ and $\mathbf{T} 2$; for example, $\hat{\phi}_{\mathrm{c}}=\hat{\varepsilon}_{\mathrm{x}}$ provides a gradient along $\hat{\mathbf{x}}$ at T0. Control potentials of the second category are designed to provide zero gradients and only curvatures at T0, whereas we require related gradients and curvatures to be zero at T1 and T2. For example, we design $\hat{\phi}_{c}=\hat{\kappa}_{\text {tune }}$, with the following non-zero constrains $\left.\partial_{y} \partial_{y} \hat{\kappa}_{\text {tune }}(\mathbf{r})\right|_{\mathbf{r}=\mathbf{T} 0}=-\left.\partial_{z} \partial_{z} \hat{\kappa}_{\text {tune }}(\mathbf{r})\right|_{\mathbf{r}=\mathbf{T} 0}=0.937 \times 10^{7} \mathrm{~m}^{-2}$ with corresponding $U_{\mathrm{c}}=U_{\text {tune. }}$. Linear combination of multiple control potentials enable us, for example, to locally compensate stray potentials up to second order, to independently control mode frequencies and orientations at each trap site, and, when implementing time-dependent control potentials, to apply directed and phase-controlled mode-frequency modulations or mode excitations.

Optical setup and experimental procedures. We employ eight laser beams at wavelengths near $280 \mathrm{~nm}$, from three distinct laser sources $^{46}$, with wave vectors parallel to the $x y$ plane (Fig. 1b) for preparation, manipulation and detection of electronic and motional states of ${ }^{25} \mathrm{Mg}^{+}$ions. Five distinct $\sigma^{+}$-polarized beams (two for Doppler cooling, two for optical pumping and one for state detection) are superimposed, with wave vector $\mathbf{k}_{\mathrm{P} / \mathrm{D}}$ (preparation/detection) aligned with a static homogeneous magnetic quantization field $\mathbf{B}_{\mathbf{0}} \simeq 4.65 \mathrm{mT}$ (Fig. 1b). The beam waists (half width at $1 / e^{2}$ intensity) are $\simeq 150 \mu \mathrm{m}$ in the $x y$ plane and $\simeq 30 \mu \mathrm{m}$ in $z$ direction, to ensure reasonably even illumination of all three trap sites, while avoiding excessive clipping of the beams on the trap chip. The two Doppler-cooling beams are detuned by $\Delta \simeq-\Gamma / 2$ and $-10 \Gamma$ (for initial Doppler cooling and state preparation by optical pumping) with respect to $|\downarrow\rangle \equiv\left|S_{1 / 2}, F=3, m_{\mathrm{F}}=+3\right\rangle \leftrightarrow\left|P_{3 / 2}, F=4, m_{\mathrm{F}}=+4\right\rangle$ with a natural line width $\Gamma /(2 \pi) \simeq 42 \mathrm{MHz}$. The state detection beam is resonant with this cycling transition and discriminates $|\downarrow\rangle$ from $|\uparrow\rangle \equiv\left|S_{1 / 2}, F=2, m_{\mathrm{F}}=+2\right\rangle$, the pseudo-spin states $|\downarrow\rangle$ and $|\uparrow\rangle$ are separated by $\omega_{0} /(2 \pi) \simeq 1,681.5 \mathrm{MHz}$. The resulting fluorescence light is collected with high numerical aperture lens onto either a photomultiplier tube or an electron-multiplying charge-coupled device camera. We prepare (and repump to) $|\downarrow\rangle$ by two optical-pumping beams that couple $|\uparrow\rangle$ and $\left|S_{1 / 2}, F=3, m_{\mathrm{F}}=+2\right\rangle$ to states in $\left|P_{1 / 2}\right\rangle$ from where the electron decays back into the ground state manifold and population is accumulated in $|\downarrow\rangle$. We can couple $|\downarrow\rangle$ to $|\uparrow\rangle$ via two-photon stimulated-Raman transitions ${ }^{25,47,48}$, while we can switch between two different beam configurations labelled $\mathrm{BR}^{*}+\mathrm{RR}$ with $\Delta \mathbf{k}_{\mathrm{x}} \| \hat{\mathbf{x}}$ and $\mathrm{BR}+\mathrm{RR}$ with $\Delta \mathbf{k}_{\mathrm{y}} \| \hat{\mathbf{y}}$. The beam waists are $\simeq 30 \mu \mathrm{m}$ in the $x y$ plane and $\simeq 30 \mu \mathrm{mm}$ in $z$ direction.

We load ions by isotope-selective photoionization from one of three atomic beams collimated by $4 \mu \mathrm{m}$ loading holes located beneath each trap site (Fig. 1). We can also transfer ions from one site to any neighbouring site via the ancillary trap by applying suitable potentials to control electrodes and a metallic mesh (with high optical transmission) located $\simeq 7 \mathrm{~mm}$ above the surface. Typically, experiments start with $2 \mathrm{~ms}$ of Doppler cooling, optionally followed by resolved sideband cooling, and $|\downarrow\rangle$ preparation via optical pumping. We use 30 channels of a 36-channel arbitrary waveform generator with $50 \mathrm{MHz}$ update rate $^{49}$ to provide static (persistent over many experiments) and dynamic (variable within single experiments) control potentials. Each experiment is completed by a pulse for pseudo-spin detection of duration $\simeq 150 \mu$ s that yields $\simeq 12$ counts on average for an ion in $|\downarrow\rangle$ and $\simeq 0.8$ counts for an ion in $|\uparrow\rangle$. Specific experimental sequences are repeated 100-250 times.
Initially, we calibrate three (static) control potentials $\hat{\varepsilon}_{\mathrm{x}}, \hat{\varepsilon}_{\mathrm{y}}$ and $\hat{\varepsilon}_{z}$ to compensate local stray fields ${ }^{50}$ with a single ion near T0, whereas we observe negligible effects on the local potentials near T1 and T2 (Methods). Rotated versions of these control potentials are used to compensate local stray fields near T1 and T2. Near each site, we achieve residual stray field amplitudes $\leq 3 \mathrm{~V} \mathrm{~m}^{-1}$ in the $x y$ plane and $\leq 900 \mathrm{~V} \mathrm{~m}^{-1}$ along $z$, currently limited by our methods for detection of micromotion.

With the stray fields approximately compensated, we characterize the trap near T0 with a single ion (Methods). We find mode frequencies of $\omega_{1} /(2 \pi) \simeq 5.3 \mathrm{MHz}, \omega_{2} /(2 \pi) \simeq 2.6 \mathrm{MHz}$ and $\omega_{3} /(2 \pi) \simeq 4.1 \mathrm{MHz}$ with frequency drifts of about $2 \pi \times 0.07 \mathrm{kHz}$ $(60 \mathrm{~s})^{-1}$; mode frequencies and orientations are altered by local stray curvatures on our chip, in particular, $\mathbf{u}_{1}$ and $\mathbf{u}_{3}$ are rotated in the $x z$ plane, while $\mathbf{u}_{2}$ remains predominantly aligned along $y$. We obtain heating rates for the modes $\mathbf{u}_{1}$ of $0.9(1)$ quanta $\mathrm{ms}^{-1}$, $\mathbf{u}_{2}$ of 2.2(1) quanta $\mathrm{ms}^{-1}$ and $\mathbf{u}_{3}$ of $4.0(3)$ quanta $\mathrm{ms}^{-1}$

Control of mode configurations at individual trap sites. The ability to control mode frequencies and orientations at each site with minimal effect on local trapping potentials at neighbouring sites is essential for the static and dynamical tuning of inter-ion Coulomb couplings. We experimentally demonstrate individual mode-frequency control using $\hat{\kappa}_{\text {tune }}$. To this end we measure local mode frequencies with a single ion near T0 or T2 (Methods). Tuning of about $\pm 2 \pi \times 80 \mathrm{kHz}$ of $\omega_{2}$ near T0 is shown in Fig. 2 as blue data points, accompanied by residual changes of about $\mp 2 \pi \times 1 \mathrm{kHz}$ in the corresponding mode frequency near the neighbouring site $\mathbf{T} 2$, depicted by red data points. To infer local control curvatures, we describe the expected detuning $\Delta \omega_{2}$ due to $\hat{\kappa}_{\text {tune }}$ at T0 (analogously at T2) by

$$
\Delta \omega_{2}=\sqrt{\omega_{2}^{2}+U_{\text {tune }}\left(\left.\frac{Q}{m} \partial_{y} \partial_{y} \hat{\kappa}_{\text {tune }}(\mathbf{r})\right|_{\mathbf{r}=\mathbf{T} \mathbf{0}}\right)}-\omega_{2},
$$

where we neglect a small misalignment of $\mathbf{u}_{2}$ from $y$. The prediction of equation (4) is shown as a blue/dashed line in Fig. 2. The blue/solid line results from a fit with a function of the form of equation (4) to the data yielding a control curvature of $1.164(3) \times 10^{7} \mathrm{~m}^{-2}$. The inset magnifies the residual change in frequency near T2. Here, a fit (red/solid line) reveals a curvature of $-0.012(2) \times 10^{7} \mathrm{~m}^{-2}$. Residual ion displacements of $\Delta z=-2.95(3) \mu \mathrm{m}$ from $\mathrm{T0}$ and $\Delta z=-2.9(4) \mu \mathrm{m}$ from $\mathrm{T} 2$, respectively, suffice to explain deviations between experimentally determined and designed curvature values and are below our current limit of precision locating the ions in that direction. In future experiments, curvature measurements may be used to further reduce stray fields.

We also implement a dynamic $U_{\text {tune }}(t)$, to adiabatically tune $\omega_{2}$ near T0 within single experiments: we prepare our initial state by Doppler cooling, followed by resolved sideband cooling of mode $\mathbf{u}_{2}$ to an average occupation number $\bar{n}_{2} \simeq 0.1$ and optical pumping to $|\downarrow\rangle$. In a next step, we apply a first adiabatic ramp from $U_{\text {tune, } \mathrm{A}}=0 \mathrm{~V}$ to $U_{\text {tune, } \mathrm{B}}$ between 0 and $2.3 \mathrm{~V}$ (corresponding to a measured frequency difference $\Delta \omega_{2} /(2 \pi) \simeq 430 \mathrm{kHz}$ ) within $t_{\text {ramp }}=7.5$ to $120 \mu \mathrm{s}$ and, subsequently, couple $|\downarrow\rangle$ and $|\uparrow\rangle$ to mode $\mathbf{u}_{2}$ with pulses of $\mathrm{BR}+\mathrm{RR}$ tuned to sideband transitions that either add or subtract a single quantum of motion. If the ion is in the motional ground state, no quantum can be subtracted and the spin state remains unchanged when applying the motion subtracting sideband pulse. The motion-adding sideband can always be driven, and comparing the spin-flip probability of the two sidebands allows us to determine the average occupation of the dynamically tuned mode ${ }^{48}$. We find that the average occupation numbers are independent of the duration of the ramp and equal to those obtained by remaining in a static 


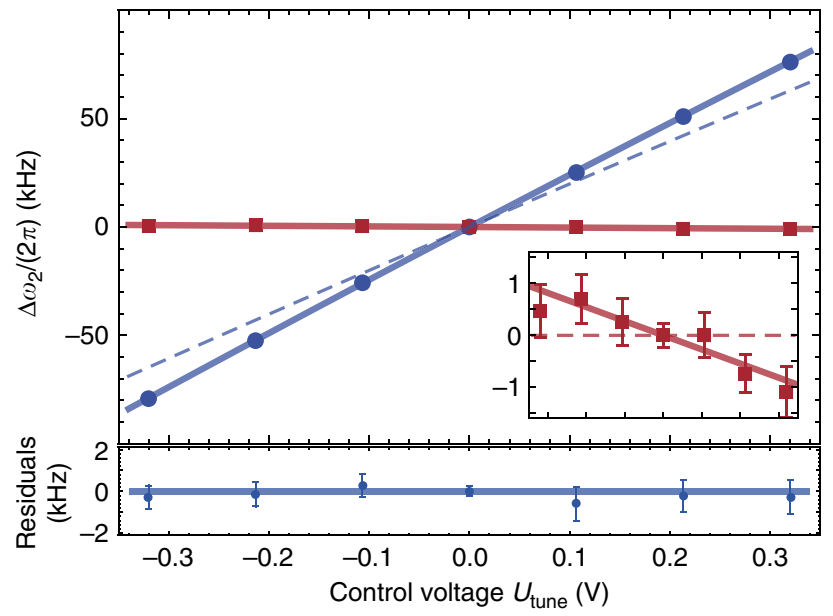

Figure 2 | Individual control of mode frequencies. Frequency change $\Delta \omega_{2} /(2 \pi)$ probed with a single ion near TO (blue dots) as a function of $U_{\text {tune }}$ The intended control curvature $0.937 \times 10^{7} \mathrm{~m}^{-2}$ (along the $y$ direction) yields the blue/dashed line (cp. equation (4)), while a fit to the data (blue/solid line) returns a control curvature of $1.164(3) \times 10^{7} \mathrm{~m}^{-2}$ (corresponding residuals shown in the bottom graph). The remaining change of the corresponding mode frequency of a single ion near T2 (red squares) is shown in the inset, for the full range of $U_{\text {tune. }}$ A fit to these data (red/solid lines) results in a residual control curvature of

$-0.012(2) \times 10^{7} \mathrm{~m}^{-2}$. Ideally, $\hat{\kappa}_{\text {tune }}$ would create no additional curvature at $\mathbf{T 2}$ (red/dashed line). We attribute the difference between designed and measured values to residual ion displacements from T0 and T2. Each data point represents the average of 250 experiments with error bars (for some data smaller than symbols) denoting the s.e.m. Residual variations of experimental parameters, for example, changes of stray potentials, can result in day-to-day variations of measurement outcomes that require recalibration to remain within our stated statistical uncertainties.

potential for $t_{\text {ramp, }}$, that is, the motion is unaffected by the dynamic tuning.

We rotate mode orientations near T0 in the $x y$ plane with a control-potential $\hat{\kappa}_{\text {rot }}$, while setting additional constraints to keep gradients and curvatures of the local trapping potential constant at T1 and T2 (Methods). We determine the rotation of mode orientations from electron-multiplying charge-coupled device images of two ions near T0 that align along $\mathbf{u}_{2}$ (axis of weakest confinement). Simultaneously, we trap one or two ions near T1 and $\mathbf{T} 2$ to monitor residual changes in ion positions and mode orientations (and frequencies) because of unwanted local gradients and curvatures of $\hat{\kappa}_{\text {rot }}$. We take 14 images for five different $\hat{\kappa}_{\text {rot }}$ values, while constantly Doppler cooling all ions and exciting fluorescence. Figure $3 \mathrm{a}$ shows two images for $U_{\text {rot }}=0 \mathrm{~V}$ (left) and $U_{\text {rot }}=2.45 \mathrm{~V}$ (right). Schematics of control electrodes are overlaid to the images and coloured to indicate their bias voltages $U_{\text {rot }}$. Ion positions (in the $x y$ plane) are obtained with an uncertainty of $\pm 0.5 \mu \mathrm{m}$, yielding uncertainties for inferred angles $\varphi_{2, y}$ of $\pm 5^{\circ}$. Here, $\varphi_{2, y}$ denotes the angle between local mode $\mathbf{u}_{2}$ and $y$. Figure $3 \mathrm{~b}$ shows measured $\varphi_{2, y}$ for ions near T0 (blue dots) and T1 (red squares) and compares them with our theoretical expectation (solid lines), further described in the Methods. We tune $\varphi_{2, y}$ between $0^{\circ}$ and $45^{\circ}$ near $\mathbf{T} \mathbf{0}$, enabling us to set arbitrary mode orientations in the $x y$ plane, whereas ion positions (mode orientations) near $\mathbf{T} 1$ and $\mathbf{T} 2$ remain constant within $\pm 0.5 \mu \mathrm{m}$ (better than $\pm 5^{\circ}$ ) in the $x y$ plane.

A complementary way of characterizing mode orientations and frequencies, now with respect to $\Delta \mathbf{k}_{x}$ and/or $\Delta \mathbf{k}_{y}$ is to analyse the probability of finding $|\downarrow\rangle$ after applying $|\downarrow\rangle \leftrightarrow|\uparrow\rangle$ (carrier) or motional sideband couplings for variable duration. If all modes of a
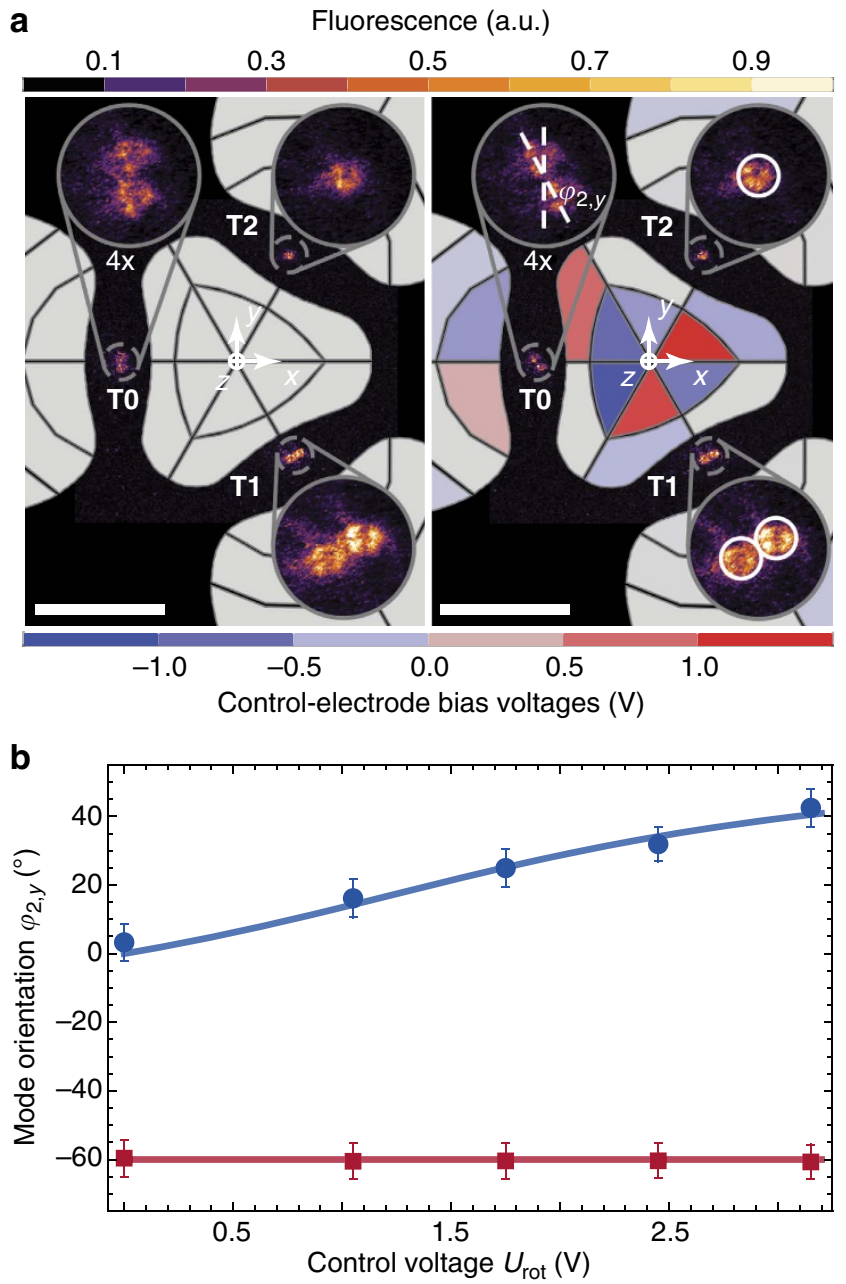

Figure 3 | Individual control of mode orientations. (a) Electronmultiplying charge-coupled device images of pairs of ions near T0 and T1 and a single ion near T2; white scale bars are $50 \mu \mathrm{m}$ and blow ups of each site are magnified by four. Schematics of control electrodes are coloured according to their bias voltage $\boldsymbol{U}_{\text {rot. }}$ lon pairs align along $\mathbf{u}_{2}$, the lowestfrequency mode. The left image captures ion positions for $U_{\text {rot }}=0 \mathrm{~V}$ and, here, $\omega_{2} /(2 \pi)=1.9(2) \mathrm{MHz}$ near To. The right image illustrates the rotation effect for $U_{\text {rot }}=2.45 \mathrm{~V}$ : Mode $\mathbf{u}_{2}$ near TO with $\omega_{2} /(2 \pi)=$ $1.8(2) \mathrm{MHz}$ is rotated by $\varphi_{2, y}=31(5)^{\circ}$, whereas ion positions near $\mathbf{T 1}$ and T2 remain unchanged; white circles indicate initial ion positions (for $U_{\text {rot }}=0$ ). (b) Mode $\mathbf{u}_{2}$ orientation in the $x y$ plane for TO (blue dots) and T1 (red squares), described by $\varphi_{2, y}$, derived from a total of 14 images as a function of $U_{\text {rot }}$; error bars denote our systematic uncertainty. The data are in good agreement with our predictions of the effect of $\hat{\kappa}_{\text {rot }}$ (solid lines).

a single ion are prepared in their motional ground state, the ratio of Rabi frequencies of carrier and sideband couplings is given by the Lamb-Dicke parameter ${ }^{48}$, which is for $\mathbf{u}_{1}$ and $\mathbf{\Delta} \mathbf{k}_{x}$ :

$$
\eta_{1, x}=\Delta \mathbf{k}_{x} \cdot \mathbf{u}_{1} \sqrt{\frac{\hbar}{2 m \omega_{1}}}=\left|\Delta \mathbf{k}_{x}\right| \sqrt{\frac{\hbar}{2 m \omega_{1}}} \cos \left(\varphi_{1, x}\right),
$$

where $\varphi_{1, x}$ is the angle between $\mathbf{u}_{1}$ and $\Delta \mathbf{k}_{x}$. The differences of carrier and sideband transition frequencies reveal the mode frequencies, whereas ratios of sideband and carrier Rabifrequencies determine Lamb-Dicke parameters and allow for finding the orientation of modes.

We use a single ion near T0 to determine the orientations and frequencies of two modes relative to $\Delta \mathbf{k}_{x}$. We apply another control potential $\hat{\kappa}_{\text {rot2 }}$, designed to rotate $\mathbf{u}_{1}$ and $\mathbf{u}_{3}$ in the $x z$ 

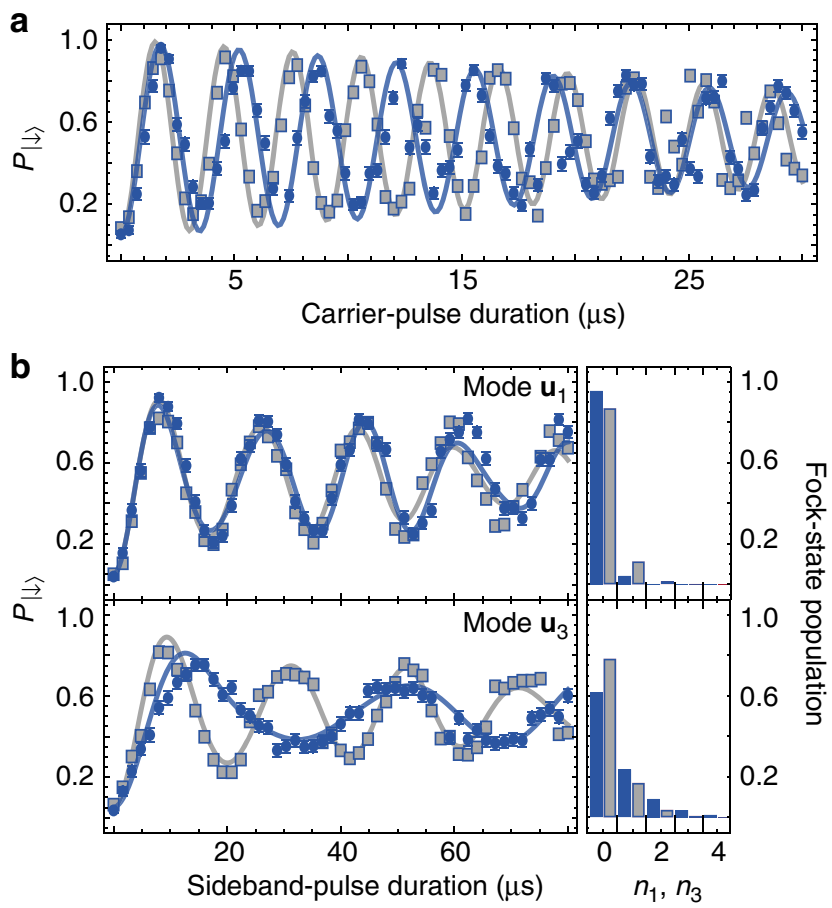

Figure 4 | Measuring mode orientations via laser couplings. Probability of $|\downarrow\rangle$ after applying carrier and sideband couplings via $\Delta \mathbf{k}_{x}$ with a single ion

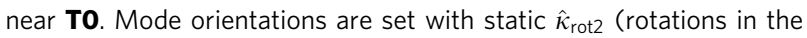
$x z$ plane) for $U_{\text {rot2 } 2}=1.62 \mathrm{~V}$ (blue dots) and $-2.43 \mathrm{~V}$ (grey squares). (a) Shows the carrier transitions, $\left|\uparrow, n_{1}, n_{3}\right\rangle \leftrightarrow\left|\downarrow, n_{1}, n_{3}\right\rangle$, whereas $\mathbf{b}$ represents the $\mathbf{u}_{1}$-sideband transitions, $\left|\uparrow, n_{1}, n_{3}\right\rangle \leftrightarrow\left|\downarrow, n_{1}+1, n_{3}\right\rangle$, and the $\mathbf{u}_{3}$-sideband transitions, $\left|\uparrow, n_{1}, n_{3}\right\rangle \leftrightarrow\left|\downarrow, n_{1}, n_{3}+1\right\rangle$. From combined model

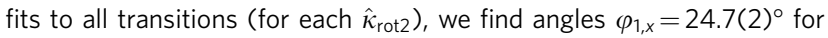
$U_{\text {rot2 }}=-1.62 \mathrm{~V}$ (blue lines) and $36.1(2)^{\circ}$ for $U_{\text {rot } 2}=-2.43 \mathrm{~V}$ (grey lines) of mode $\mathbf{u}_{1}$ relative to $\Delta \mathbf{k}_{x}$. Histograms in $\mathbf{b}$ display derived Fock-state populations with thermal average occupation numbers between $\simeq 0.05$ and $\simeq 0.6$. Each data point is the average of 250 experiments and error bars (for some data smaller than symbols) denote the s.e.m. Residual variations of experimental parameters, for example, changes of stray potentials, can result in day-to-day variations of measurement outcomes that require recalibration to remain within our stated statistical uncertainties.

plane near T0, and implement carrier and sideband couplings to both modes with $\Delta \mathbf{k}_{x}$ after resolved sideband cooling and initializing $|\uparrow\rangle$. In Fig. 4, the probability of $|\downarrow\rangle$ is shown for different pulse durations of carrier couplings (top) and sideband couplings to mode $\mathbf{u}_{1}$ (middle) and $\mathbf{u}_{3}$ (bottom). Data points for $U_{\text {rot2 }}=-1.62 \mathrm{~V}$ are shown as blue rectangles and for $-2.43 \mathrm{~V}$ as grey rectangles. We fit each data set to a theoretical model (blue and grey lines) to extract the angles ${ }^{51}$ and distributions of Fockstate populations of each mode (shown as histograms): we find $\varphi_{1, x}=24.7(2)^{\circ}$ for $U_{\text {rot } 2}=-1.62 \mathrm{~V}$ and $\varphi_{1, x}=36.1(2)^{\circ}$ for $U_{\text {rot2 }}=-2.43 \mathrm{~V}$, whereas average occupation numbers range between $\simeq 0.05$ and $\simeq 0.6$. Adding measurements along $\Delta \mathbf{k}_{y}$ and taking into account that the normal modes have to be mutually orthogonal would allow to fully reconstruct all mode orientations. With resolved sideband cooling on all three modes, we can prepare a well-defined state of all motional DoF.

\section{Discussion}

We characterized two trap arrays that confine ions on the vertices of equilateral triangles with side lengths 80 and $40 \mu \mathrm{m}$. We developed systematic approaches to individually tune and calibrate control potentials in the vicinity of each trap site of the $80-\mu \mathrm{m}$ array, by applying bias potentials to 30 control electrodes. With suitably designed control potentials, we demonstrated precise individual control of mode frequencies and orientations. By utilizing a multi-channel arbitrary waveform generator, we also dynamically changed control potentials within single experimental sequences without adverse effects on spin or motional states. Further, we devised a method to fully determine all mode orientations (and frequencies) based on the analysis of carrier and sideband couplings. Measured heating rates are currently comparable to the expected inter-ion Coulomb coupling rate of $\Omega_{\text {ex }} /(2 \pi) \simeq 1 \mathrm{kHz}$ for ${ }^{25} \mathrm{Mg}^{+}$ions in the $40-\mu \mathrm{m}$ array at mode frequencies of $\simeq 2 \pi \times 2 \mathrm{MHz}$ (ref. 32). This coupling rate sets a fundamental time scale for effective spin-spin couplings ${ }^{33}$. To observe coherent spin-spin couplings, ambient heating needs to be reduced. Decreases in heating rates of up to two orders of magnitude would leave $\Omega_{\mathrm{ex}}$ considerably higher than competing decoherence rates and allow for coherent implementation of fairly complex spin-spin couplings. Such heating rate reductions have been achieved in other surface traps by treatments of the electrode structure s4-36 $^{3}$ and/or cryogenic cooling of the electrodes ${ }^{37-39}$. The couplings in question have been observed in one dimension in a cryogenic system ${ }^{32,33}$.

Currently, we can compensate stray fields, set up normal mode frequencies and directions for all three ions and initialize them for a two-dimensional AQS, that is, prepare a fiducial initial quantum state for ions at each trap site. A complete AQS may use the sequence presented in Fig. 5. A dynamic ramp adiabatically transforms the system between two control sets, labelled as A and $B$, that realize specific mode frequencies and orientations at each site. Set A may serve to globally initialize spin-motional states of ions, potentially with more than one ion at each site, that could be the ground state of a simple initial Hamiltonian. At all sites, mode frequencies and orientations need to be suitable (bottom left of Fig. 5) to enable global resolved sideband cooling, ideally preparing ground states for all motional modes. A first ramp to set B combined with appropriate laser fields may be used to adiabatically or diabatically realize a different Hamiltonian, for example, by turning on complex spin-spin couplings. Mode frequencies and orientations are tuned such that the Coulomb interactions between ions can mediate effective spin-spin couplings, for example, all mode vectors $\mathbf{u}_{1}$ are rotated to point to the centre of the triangle (bottom right of Fig. 5). During the application of such interactions, the ground state of the uncoupled system can evolve into the highly entangled ground state of a complex coupled system. In contrast, diabatic ramping to set $\mathrm{B}$ will quench the original ground state and the coupled system will evolve into an excited state that is not an eigenstate. After a final adiabatic or diabatic ramp back to set $\mathrm{A}$, we can use global (or local) laser beams to read out the final spin states at each site.

In this way, our arrays may become an arbitrarily configurable and dynamically reprogrammable simulator for complex quantum dynamics. It may enable, for example, the observation of photonassisted tunnelling, as required for experimental simulations of synthetic gauge fields ${ }^{52,53}$ or other interesting properties of finite quantum systems, such as thermalization, when including the motional $\mathrm{DoF}^{54}$. Concentrating on spin-spin interactions, the complex entangled ground states of spin frustration can be studied in the versatile testbed provided by arrays of individually trapped and controlled ions ${ }^{30,55}$. Arrays with a larger number of trap sites could realize a level of complexity impossible to simulate on conventional computers ${ }^{56,57}$.

\section{Methods}

Design of arrays used in the experiments. The design of arrays used in the expeiments is based on the methods described in ref. 29. In particular, we use the Mathematica package for surface atom and ion traps ${ }^{43}$ to globally optimize the RF 
electrode shape for maximal curvature with a given amplitude of the RF drive, whereas producing smooth continuous electrode shapes that require a single RF drive to operate the array. We specify the desired trap site positions as well as the ratio and orientation of normal-mode frequencies as a fixed input to the optimization algorithm for the pseudopotential, that is, we define that the high-

a

Set $B$ to Set A

\begin{tabular}{lcc}
$\begin{array}{l}\text { Initial-state } \\
\text { preparation }\end{array}$ & $\begin{array}{c}\text { Implement } \\
\text { inter-ion couplings }\end{array}$ & $\begin{array}{c}\text { Final-state } \\
\text { detection }\end{array}$ \\
\hline & & Sequence duration
\end{tabular}

b Set A

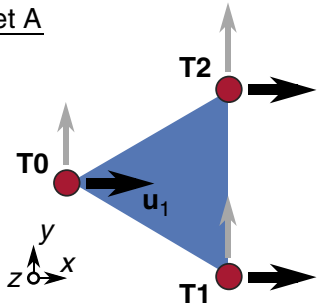

Set B

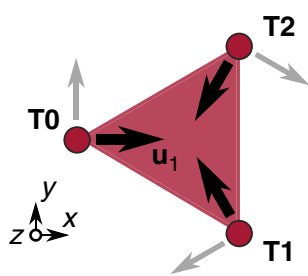

Figure 5 | Generic experimental sequence for tuneable inter-ion couplings. (a) Time-line of an experiment starting with the initialization of an fiducial quantum state of all ions in the array, followed by an adiabatic (or diabatic) ramp of control potentials between sets $A$ and $B$ that reconfigure the normal-mode structure from the setup, see $\mathbf{b}$. Then, appropriate laser fields implement inter-ion couplings required for the AQS. The simulation completes with ramping the control potentials back to set $A$, where the individual spin states can be detected. (b) Examples for configurations of motional DoF are illustrated by the arrows that show the orientation of $\mathbf{u}_{1}$ at the three trap sites (red dots). Set A may be applied when globally and/or locally preparing and detecting spin-motional states, whereas set B can establish specific inter-ion Coulomb couplings to mediate, for example, effective spin-spin couplings for AQS, cp. refs 33,55. frequency mode (for all three sites) lies within the $x y$ plane and points towards the virtual centre of the array. Resulting electrode regions held to ground are subdivided into separated control electrodes that provide complete and independent control over the eight $\mathrm{DoF}$ at each site.

Array scaling for future realisations. To ensure that our approach can be scaled to more than three trapping sites, we compare designs of arrays containing different numbers of sites, $N_{\text {sites }}$, that are optimized by the algorithm described in ref. 29. Here, we assume a fixed ratio of $h / d=1 / 2$, where $h$ denotes the distance of the sites to the nearest electrode surface and $d$ is the inter-site distance. Further, we specify for all arrays that the high-frequency mode is aligned orthogonally to the $x y$ plane at each site, in contrast to our demonstrated arrays (see Fig. 1 for details). This unique mode configuration permits a fair comparison of geometries with increasing $N_{\text {sites. }}$. To illustrate the optimal electrode shapes, we present four examples of triangular arrays with $N_{\text {sites }}=\{3,6,18,69\}$ in Fig. $6 \mathrm{a}-\mathrm{d}$. To enable the same level of individual control as demonstrated for both of our three-site arrays we would have to subdivide the optimized ground electrodes into $\geq 8 \times N_{\text {sites }}$ control electrodes. We find that the inner areas converge to fairly regular electrode shapes for larger $N_{\text {sites, }}$, whereas electrodes closer to the border are deformed to compensate for edge effects (see Fig. $6 \mathrm{~d}$ for details). However, the spatial extent and complexity of all electrodes remains comparable to the arrays used in our experiments and, thus, fabrication of these larger arrays can be accomplished by scaling the applied techniques (see below).

To quantify the geometric strength of individual trap sites independently of $m$, $U_{\mathrm{RF}}, \Omega_{\mathrm{RF}}$ and $h$, we consider the dimensionless curvature $\kappa$ of the pseudopotential that we normalize to the highest possible curvature for a single $\operatorname{site}^{29}$. We show optimized $\kappa$ for arrays with $N_{\text {sites }}$ between 1 and 102, as well as, the value for $N_{\text {sites }}=\infty$ in Fig. 6e; a fully controlled array with $N_{\text {sites }}=102$ should be sufficient to study quantum many-body dynamics that are virtually impossible to simulate on a conventional computer. We find that $k$ for $N_{\text {sites }}=102$ is reduced by about a factor of two compared with $\kappa \simeq 0.87$ for $N_{\text {sites }}=3$, whereas $\kappa \simeq 0.07$ for $N_{\text {sites }}=\infty$; see ref. 29 for a detailed discussion of infinite arrays. The decrease in trap curvature can be compensated in experiments by adjusting $U_{\mathrm{RF}}$ and $\Omega_{\mathrm{RF}}$ correspondingly, or by reducing $h$. Further, we estimate that trapping depths remain on the same order of magnitude for increasing $N_{\text {sites }}$ compared with our demonstrated arrays (cp. Fig. 1d). For an infinite array it has been shown that depths of a few $\mathrm{mV}$ are achievable ${ }^{30}$. Note, that in surface-electrode traps the trapping potential is less deep along $z$ than in the $x y$ plane, and ion-escape points (closest and lowest saddle point of the pseudopotential) typically lie above each site. In experiments, we may apply a constant bias potential to the control electrodes, surrounding ground planes, and the mesh (cover plane) to increase the depth along $z$ to a level where trapping is a<smiles></smiles>

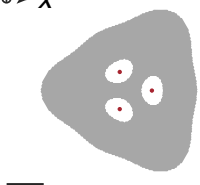

d

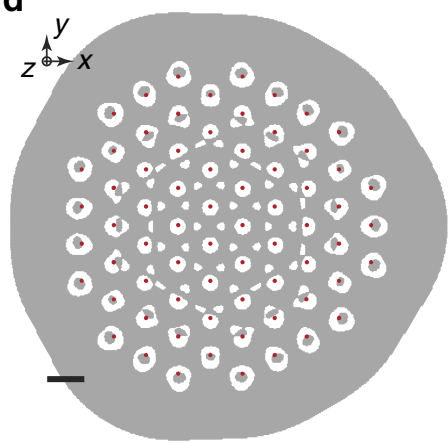

b

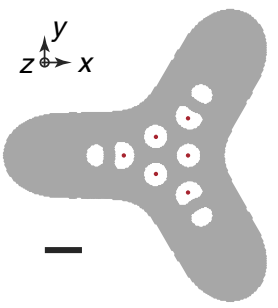

C

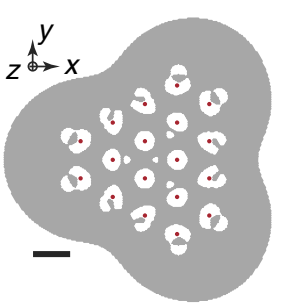

e

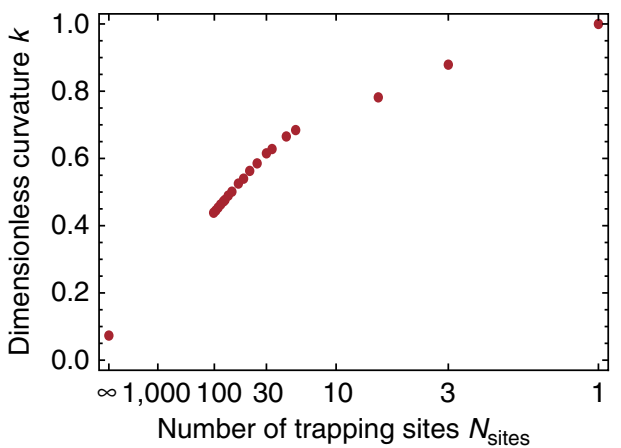

Figure 6 | Scale-up the number of sites in triangular arrays. (a-d) Optimized surface-electrode shapes for 3, 6, 18 and 69 trapping sites, where we assume $h / d=1 / 2$ (as for our $80 \mu \mathrm{m}$ array); black scale bars have fixed length $d$. The high-frequency mode is aligned orthogonally to the $x y$ plane in each trapping site, to permit comparability of the different geometries. RF and ground electrodes are coloured in grey and white, respectively, and individual trap sites are marked with red dots. In a functional array, we will have to further subdivide the ground electrodes to enable individual control at each site, as demonstrated in our work with three sites. (e) Optimized dimensionless curvature $\kappa$ as a function of the number of trapping sites in the triangular arrays, quantifying the strength of the individual traps. For a single site $\kappa=1.0$ and for an infinite triangular lattice $\kappa \simeq 0.07$. In experimental realizations, we can compensate this decrease in trap curvature by adjusting $U_{\mathrm{RF}}$ and $\Omega_{\mathrm{RF}}$ or by reducing $h$. 
routinely achieved, while reducing the depth in the $x y$ plane $^{30}$. With such measures in place, we are fairly confident that ions created by photoionization from a hot atomic beam can be loaded and cooled into the local minima of larger arrays.

Architecture of our trap chip. The $10 \times 10 \mathrm{~mm}^{2} \mathrm{Si}$ substrate of our trap chip is bonded onto a $33 \times 33 \mathrm{~mm}^{2}$ ceramic pin grid array $(\mathrm{CPGA})$; the electrodes of the trap arrays are wire-bonded with aluminium wires to the pins of the CPGA, with independent pins for the RF electrodes of the two arrays. The trap chip contains four aluminum- $1 / 2 \%$ copper metal layers, that are electrically connected by tungsten vertical interconnects thereby allowing 'islanded' control electrodes in the top electrode layer (Fig. 1). The buried electrical leads are isolated by intermediate $\mathrm{SiO}_{2}$ layers, nominally $2 \mu \mathrm{m}$ thick, while the surface layer is spaced by $10 \mu \mathrm{m}$ from the buried layers. All electrodes are mutually separated by nominally $1.2-1.4 \mu \mathrm{m}$ gaps and a $50-$ $\mathrm{nm}$ gold layer is evaporated on the top surfaces in a final fabrication step. The trap chip fabrication is substantially the same as that described in the Supplement to ref. 40. Each control electrode is connected to ground by $820 \mathrm{pF}$ capacitors located on the CPGA to minimize potential changes due to capacitive coupling to the RF electrodes.

Compensation of stray potentials at each site. For compensation of local stray fields in the $x y$ plane, we vary the strength of individual control potentials $\hat{\varepsilon}_{\mathrm{x}}$ and $\hat{\varepsilon}_{\mathrm{y}}$ and find corresponding coefficient settings where we obtain a maximal Rabi rate of the detection transition and/or minimal Rabi rates of micromotion-sideband transitions probed with $\Delta \mathbf{k}_{\mathrm{x}}$ and $\Delta \mathbf{k}_{\mathrm{y}}$; resulting in residual stray-field amplitudes of $\leq 3 \mathrm{~V} \mathrm{~m}^{-1}$. For compensation along $z$, we vary the strength of individual $\hat{\varepsilon}_{\mathrm{z}}$ to minimize a change in ion position due to a modulation of $U_{\mathrm{RF}}$. The depth of field of our imaging optics aids to detect changes in $z$-position via blurring of images of single ions trapped at each site, within an uncertainty of about $\pm 5 \mu \mathrm{m}$. This corresponds to residual stray-field amplitudes of $\simeq 900 \mathrm{~V} \mathrm{~m}^{-1}$ for typical trapping parameters.

Mode frequency and heating rate measurements. To measure mode frequencies, we Doppler-cool the ion and pump to $|\downarrow\rangle$. Then, we apply a motional excitation pulse with fixed duration $t_{\mathrm{exc}}=100 \mu \mathrm{s}$ to a single control electrode. The pulse produces an electric field oscillating at a frequency $\omega_{\text {exc }}$ that excites the motion, if $\omega_{\text {exc }}$ is resonant with a mode frequency, and we can detect mode amplitudes of $>100 \mathrm{~nm}$ along $\mathbf{k}_{\mathrm{D}}$ via the Doppler effect. In the experiments, we vary $\omega_{\text {exc }}$ and obtain resonant excitations at $\omega_{j}$ with $j=\{1,2,3\}$. By repeating measurements, we record $\simeq 50$ consecutive frequency values for each mode frequency over the course of $\Delta t \simeq 1 \mathrm{~h}$ with a single ion near T0. The results are consistent with linear changes in frequencies, with rates $\Delta \omega_{1} / \Delta t=$ $-2 \pi \times 0.090(3) \mathrm{kHz}(60 \mathrm{~s})^{-1}, \Delta \omega_{2} / \Delta t=-2 \pi \times 0.064(1) \mathrm{kHz}(60 \mathrm{~s})^{-1}$ and $\Delta \omega_{3} / \Delta t=-2 \pi \times 0.063(5) \mathrm{kHz}(60 \mathrm{~s})^{-1}$.

For the heating rate measurements, we add multiple resolved-sideband cooling pulses after Doppler cooling to our sequence and determine mode temperatures from the sideband ratios for several different delay times ${ }^{58}$. In our experiments, we either use $\Delta \mathbf{k}_{\mathrm{x}}$ to iteratively address $\mathbf{u}_{1}$ and $\mathbf{u}_{3}$ or $\Delta \mathbf{k}_{\mathrm{y}}$ to address only $\mathbf{u}_{2}$. For this, we prepare similar mode orientations as presented in Fig. 4, find initial mode temperatures after cooling to $\bar{n}_{j} \lesssim 0.3$, and obtain corresponding heating rates.

Potentials for individual control. As a representative example for designing control potentials, we discuss $\hat{\kappa}_{\text {rot }}$ that serves to rotate the normal modes in the $x y$ plane. At position T0, the constraints are:

$$
\left.\left[\partial_{k} \partial_{l}\right] \hat{\kappa}_{\text {rot }}(\mathbf{r})\right|_{\mathbf{r}=\mathbf{T} 0}=\left(\begin{array}{ccc}
-1.60 & 1.75 & 0 \\
1.75 & 0.84 & 0 \\
0 & 0 & 0.76
\end{array}\right) \times 10^{7} \mathrm{~m}^{-2},
$$

for $k$ and $l=\{x, y, z\}$, while local gradients at all three trap sites and local curvatures at $\mathbf{T} 1$ and $\mathbf{T} \mathbf{2}$ are required to be zero. We add diagonal elements in $\left.\left[\partial_{k} \partial_{l}\right] \hat{\kappa}_{\text {rot }}(\mathbf{r})\right|_{\mathbf{r}=\mathbf{T} 0}$ to reduce changes of the $\mathbf{u}_{2}$ frequency during variation of $\hat{\kappa}_{\text {rot }}$ around our initial mode configurations. The mode configurations in the real array deviate from those derived from the $\phi_{\mathrm{ps}}$ due to additional curvatures near each trap site generated by stray potentials on our chip. Ideally, we would design control potentials for mode rotations such that all frequencies stay fixed. This is only possible if we explicitly know the initial mode configuration. In addition, we keep mode vectors tilted away from $z$ to sufficiently Doppler cool all modes during state initialization. Similarly, we design $\hat{\kappa}_{\text {rot2 }}$ to rotate modes in the $x z$ plane.

Model for varying mode orientations. To model the rotation angle $\varphi_{2, y}$ of $\mathbf{u}_{2}$ near T0 as a function of $\hat{\kappa}_{\text {rot }}$, we consider the final trapping curvature at T0 (analogously for neighbouring sites):

$$
\left.\left[\partial_{k} \partial_{l}\right] \phi_{\text {fin }}\left(U_{\text {rot }}\right)\right|_{\mathbf{r}=\mathbf{T}_{0}}=\left.\left[\partial_{\mathbf{k}} \partial_{1}\right] \phi_{\text {ini }}\right|_{\mathbf{r}=\mathbf{T}_{0}},+\left.U_{\text {rot }}\left[\partial_{\mathrm{k}} \partial_{1}\right] \kappa_{\text {rot }}\right|_{\mathbf{r}=\mathbf{T}_{0}},
$$

where $\phi_{\mathrm{ini}}(\mathbf{r})$ represents the initial potential, that is, the sum of the pseudopotential, stray potential and additional control potentials (used for stray field compensation). The local curvatures (mode frequencies and vectors) of $\phi_{\text {ini }}(\mathbf{r})$ near T0 are estimated from calibration experiments. For simplicity, we reduce equation (7) to two dimensions (in the $x y$ plane) and find corresponding eigenvectors and eigenvalues for $U_{\text {rot }}$ between 0.0 and $3.0 \mathrm{~V}$. We obtain angles $\varphi_{2, y}\left(U_{\text {rot }}\right)$ of the eigenvector $\mathbf{u}_{2}$ and we show resulting values as an interpolated solid line in Fig. $3 \mathrm{~b}$.
Similarly, we model the effect of $\hat{\kappa}_{\text {tune }}$ on $\omega_{2}$. We assume that for $U_{\text {tune }}=0$, the corresponding mode vector $\mathbf{u}_{2}$ is aligned parallel to $y$. This is the case for pure RF confinement (cp. Fig. 1c) and sufficiently small stray curvatures. We design $\hat{\kappa}_{\text {tune }}$ to tune the curvature along $y$, and the curvature as a function of $U_{\text {tune }}$ (along this axis) is described by: $\left.\partial_{y} \partial_{y} \phi_{\text {fin }}\left(U_{\text {tune }}\right)\right|_{\mathbf{r}=\mathbf{T}_{0}}=\left.\partial_{y} \partial_{y} \phi_{\text {ini }}\right|_{\mathbf{r}=\mathbf{T}_{0}}+\left.\mathrm{U}_{\text {tune }} \partial_{y} \partial_{y} \hat{\kappa}_{\text {tune }}\right|_{\mathbf{r}=\mathbf{T}_{0}}$. Finally, we insert this into $\omega_{2}=\left.\sqrt{Q / m \partial_{y} \partial_{y} \phi_{\text {ini }} \mid}\right|_{\mathbf{r}=\mathrm{T}_{0}}$ to find equation (4).

Data availability. The data that support the findings of this study are available from the corresponding author upon request.

\section{References}

1. Feynman, R. P. Simulating physics with computers. Int. J. Theor. Phys. 21, 467-488 (1982).

2. Georgescu, I. M., Ashhab, S. \& Nori, F. Quantum simulation. Rev. Mod. Phys. 86, 153-185 (2014)

3. Schaetz, T., Monroe, C. R. \& Esslinger, T. Focus on quantum simulation. N. J. Phys. 15, 085009 (2013).

4. Lanyon, B. P. et al. Towards quantum chemistry on a quantum computer. Nat Chem 2, 106-111 (2010).

5. Fleming, G. R., Huelga, S. F. \& Plenio, M. B. Focus on quantum effects and noise in biomolecules. N. J. Phys. 13, 115002 (2011).

6. Aspuru-Guzik, A. \& Walther, P. Photonic quantum simulators. Nat. Phys 8, 285-291 (2012).

7. Houck, A. A., Tureci, H. E. \& Koch, J. On-chip quantum simulation with superconducting circuits. Nat. Phys 8, 292-299 (2012).

8. Bloch, I., Dalibard, J. \& Nascimbene, S. Quantum simulations with ultracold quantum gases. Nat. Phys 8, 267-276 (2012).

9. Blatt, R. \& Roos, C. F. Quantum simulations with trapped ions. Nat. Phys 8 , 277-284 (2012).

10. Cirac, J. I. \& Zoller, P. Goals and opportunities in quantum simulation. Nat. Phys 8, 264-266 (2012).

11. Leibfried, D. Could a boom in technologies trap Feynman's simulator? Nature 463, 608-608 (2010)

12. Leibfried, D. et al. Trapped-ion quantum simulator: experimental application to nonlinear interferometers. Phys. Rev. Lett. 89, 247901 (2002).

13. Friedenauer, A., Schmitz, H., Glueckert, J. T., Porras, D. \& Schaetz, T. Simulating a quantum magnet with trapped ions. Nat. Phys 4, 757-761 (2008).

14. Schmitz, H. et al. Quantum walk of a trapped ion in phase space. Phys. Rev. Lett. 103, 090504 (2009).

15. Schmitz, H. et al. The arch of simulating quantum spin systems with trapped ions. Appl. Phys. B 95, 195-203 (2009).

16. Zahringer, F. et al. Realization of a quantum walk with one and two trapped ions. Phys. Rev. Lett. 104, 100503 (2010).

17. Gerritsma, R. et al. Quantum simulation of the Dirac equation. Nature 463, 68-71 (2010).

18. Matjeschk, R. et al. Experimental simulation and limitations of quantum walks with trapped ions. N. J. Phys. 14, 035012 (2012).

19. Porras, D. \& Cirac, J. I. Effective quantum spin systems with trapped ions. Phys. Rev. Lett. 92, 207901 (2004).

20. Islam, R. et al. Emergence and frustration of magnetism with variable-range interactions in a quantum simulator. Science 340, 583-587 (2013).

21. Britton, J. W. et al. Engineered two-dimensional ising interactions in a trappedion quantum simulator with hundreds of spins. Nature 484, 489-492 (2012).

22. Blatt, R. \& Wineland, D. Entangled states of trapped atomic ions. Nature $\mathbf{4 5 3}$ 1008-1015 (2008).

23. Hauke, P., Cucchietti, F. M., Tagliacozzo., L., Deutsch, I. \& Lewenstein, M. Can one trust quantum simulators? Rep. Prog. Phys 75, 082401 (2012).

24. Seidelin, S. et al. Microfabricated surface-electrode ion trap for scalable quantum information processing. Phys. Rev. Lett. 96, 253003 (2006).

25. Wineland, D. J. et al. Experimental issues in coherent quantum-state manipulation oftrapped atomic ions. J. Res. Natl. Inst. Stand. Technol. 103, 259 (1998).

26. Kielpinski, D., Monroe, C. \& Wineland, D. J. Architecture for a large-scale iontrap quantum computer. Nature 417, 709-711 (2002).

27. Amini, J. M. et al. Toward scalable ion traps for quantum information processing. N. J. Phys. 12, 033031 (2010)

28. Schaetz, T. et al. Towards (scalable) quantum simulations in ion traps. J. Modern Opt. 54, 2317-2325 (2007).

29. Schmied, R., Wesenberg, J. H. \& Leibfried, D. Optimal surface-electrode trap lattices for quantum simulation with trapped ions. Phys. Rev. Lett. 102, 233002 (2009).

30. Schmied, R., Wesenberg, J. H. \& Leibfried, D. Quantum simulation of the hexagonal Kitaev model with trapped ions. N. J. Phys. 13, 115011 (2011).

31. Sterling, R. C. et al. Fabrication and operation of a two-dimensional ion-trap lattice on a high-voltage microchip. Nat. Commun 5, 3637 (2014).

32. Brown, K. R. et al. Coupled quantized mechanical oscillators. Nature 471, 196-199 (2011). 
33. Wilson, A. C. et al. Tunable spin-spin interactions and entanglement of ions in separate potential wells. Nature 512, 57-60 (2014).

34. Allcock, D. T. C. et al. Reduction of heating rate in a microfabricated ion trap by pulsed-laser cleaning. N. J. Phys. 13, 123023 (2011).

35. Hite, D. A. et al. 100-fold reduction of electric-field noise in an ion trap cleaned with in-situ argon-ion-beam bombardment. Phys. Rev. Lett. 109, 103001 (2012).

36. Daniilidis, N. et al. Surface noise analysis using a single-ion sensor. Phys. Rev. B 89, 245435 (2014)

37. Deslauriers, L. et al. Scaling and suppression of anomalous heating in ion traps. Phys. Rev. Lett. 97, 103007 (2006).

38. Labaziewicz, J. et al. Suppression of heating rates in cryogenic surface-electrode ion traps. Phys. Rev. Lett. 100, 013001 (2008)

39. Labaziewicz, J. et al. Temperature dependence of electric field noise above gold surfaces. Phys. Rev. Lett. 101, 180602 (2008).

40. Tabakov, B. et al. Assembling a ring-shaped crystal in a microfabricated surface ion trap. Phys. Rev. Applied 4, 031001 (2015).

41. Ghosh, P. K. Ion Traps (Clarendon, 1995)

42. Schmied, R. Electrostatics of gapped and finite surface electrodes. N. J. Phys. 12, 023038 (2010).

43. Schmied, R. SurfacePattern: a Mathematica package for surface atom and ion traps. https://atom.physik.unibas.ch/people/romanschmied/code/ SurfacePattern.php (2009-2015).

44. Hucul, D. et al. On the transport of atomic ions in linear and multidimensional ion trap arrays. Quant. Inf. Comput. 8, 501-578 (2008).

45. Blakestad, R. B. et al. Near-ground-state transport of trapped-ion qubits through a multidimensional array. Phys. Rev. A 84, 032314 (2011).

46. Friedenauer, A. et al. High power all solid state laser system near $280 \mathrm{~nm}$. Appl. Phys. B 84 (2006).

47. Monroe, C. et al. Resolved-sideband Raman cooling of a bound atom to the 3D zero-point energy. Phys. Rev. Lett. 75, 4011-4014 (1995).

48. Leibfried, D., Blatt, R., Monroe, C. \& Wineland, D. Quantum dynamics of single trapped ions. Rev. Mod. Phys. 75, 281-324 (2003).

49. Bowler, R., Warring, U., Britton, J. W., Sawyer, J. \& Amini, B. C. Arbitrary waveform generator for quantum information processing with trapped ions. Rev. Sci. Instrum 84, 033108 (2013).

50. Berkeland, D. J., Miller, J. D., Bergquist, J. C., Itano, W. M. \& Wineland, D. J. Minimization of ion micromotion in a Paul trap. J. Appl. Phys. 83, 5025-5033 (1998).

51. Kalis, H. et al. Motional-Mode Analysis of Trapped Ions. Preprint at http:// arxiv.org/abs/1605.01272 (2016).

52. Bermudez, A., Schaetz, T. \& Porras, D. Synthetic gauge fields for vibrational excitations of trapped ions. Phys. Rev. Lett. 107, 150501 (2011).

53. Bermudez, A., Schaetz, T. \& Porras, D. Photon-assisted-tunneling toolbox for quantum simulations in ion traps. N. J. Phys. 14, 053049 (2012).

54. Clos, G., Porras, D., Warring, U. \& Schaetz, T. Time-resolved observation ofthermalization in an isolated quantum system, Preprint at http://arxiv.org/ abs/1509.07712 (2015).
55. Schneider, C., Porras, D. \& Schaetz, T. Experimental quantum simulations of many-body physics with trapped ions. Rep. Prog. Phys 75, 024401 (2012).

56. Shi, T. \& Cirac, J. I. Topological phenomena in trapped-ion systems. Phys. Rev. A 87, 013606 (2013).

57. Nielsen, A. E. B., Sierra, G. \& Cirac, J. I. Local models of fractional quantum hall states in lattices and physical implementation. Nat. Commun 4, 2864 (2013).

58. Turchette, Q. A. et al. Heating of trapped ions from the quantum ground state. Phys. Rev. A 61, 063418 (2000).

\section{Acknowledgements}

This work was supported by DFG (SCHA 972/6-1). Sandia National Laboratories is a multiprogram laboratory managed and operated by Sandia Corporation, a wholly owned subsidiary of Lockheed Martin Corporation, for the US Department of EnergyÕs National Nuclear Security Administration under Contract No. DE-AC04-94AL85000. All statements of fact, opinion or analysis expressed in this paper are those of the authors and do not necessarily reflect the official positions or views of the Office of the Director of National Intelligence (ODNI) or the Intelligence Advanced Research Projects Activity. We thank J. Denter for technical assistance. Further, we are grateful for helpful comments on the manuscript given by S. Todaro, K. McCormick and Y. Minet.

\section{Author contributions}

M.M., H.K. and U.W. participated in the design of the experiment and built the experimental apparatus. M.M., H.K., M.W., F.H. and U.W. collected data and analysed results. M.M., U.W., D.L. and T.S. wrote the manuscript. R.S. and D.L. participated in the design of the trap arrays and the experiment. M.B., P.M. and D.L.M. participated in the design and fabricated the trap chips. T.S. participated in the design and analysis of the experiment. M.M. and H.K. contributed equally to this work and all authors discussed the results and the text of the manuscript.

\section{Additional information}

Competing financial interests: The authors declare no competing financial interests.

Reprints and permission information is available online at http://npg.nature.com/ reprintsandpermissions/

How to cite this article: Mielenz, M. et al. Arrays of individually controlled ions suitable for two-dimensional quantum simulations. Nat. Commun. 7:11839 doi: 10.1038/ncomms11839 (2016).

(c) (i) This work is licensed under a Creative Commons Attribution 4.0 cc. International License. The images or other third party material in this article are included in the article's Creative Commons license, unless indicated otherwise in the credit line; if the material is not included under the Creative Commons license, users will need to obtain permission from the license holder to reproduce the material. To view a copy of this license, visit http://creativecommons.org/licenses/by/4.0/ 